\title{
Industria relacional y economía de la contribución*
}

\author{
Bernard Stiegler \\ Traducción del francés al español de Luis Alfonso Paláu-Castaño \\ Universidad Nacional de Colombia, Medellín, Colombia \\ lapalau@gmail.com
}

\begin{abstract}
Se obtiene un ideal-tipo cuando se acentúa unilateralmente uno o muchos puntos de vista, y se encadena con ello una multitud de fenómenos, dados aisladamente, difusos $y$ discretos, que se encuentran ora en gran número, ora en pequeño número, $y$ en algunos lugares para nada; que se ordenan siguiendo los precedentes puntos de vista elegidos unilateralmente, para formar un cuadro de pensamiento homogéneo. En ninguna parte se encontrará empíricamente semejante cuadro en su pureza conceptual: es una utopía. El trabajo histórico tendrá por tarea determinar en cada caso particular hasta qué punto la realidad se acerca o se aleja de este cuadro ideal.
\end{abstract}

(Max Weber, 1981)

\section{Tres convicciones en cuanto al nuevo mundo industrial}

He contribuido a la iniciativa y a la concepción de estos diálogos sobre la base de tres convicciones.

La primera de ellas es que — como lo indica el título de este ciclo de encuentros- vivimos más que nunca en un mundo industrial y la calamitosa fábula de lo que se llamó la sociedad "posindustrial" está por fin detrás de nosotros; nuestra época conoce transformaciones siempre más radicales y más rápidas, y a ese proceso de innovación permanente, que constituye un fenómeno extraordinariamente nuevo y extraño desde el punto de vista de la historia, de la protohistoria y de la prehistoria de la humanidad, que se llama la modernización, es más industrial que nunca. Es la época de una industria de servicios tal que la industrialización afecta de acá en adelante la vida en su totalidad, las relaciones

Cómo citar: Stiegler, B. (2020). Industria relacional y economía de la contribución. Ciencias Sociales y Educación, 9(17), 237-250. https://doi.org/10.22395/csye.v9n17a11

Traducción realizada por Alfonso Paláu-Castaño. Agradecemos a la editorial Fayard por permitir el acceso del libro en francés para su versión parcial en español. Se conserva la presentación editorial del francés. Nota del editor.

Recibido: 10 de febrero de 2020.

Aprobado: 16 de marzo de 2020. 
sociales más diversas y la actividad psíquica hasta en sus mínimos rincones. La he llamado la época hiperindustrial (Stiegler, 2004).

Mi segunda convicción es que cambiamos de mundo industrial. El que dejamos atrás reposaba sobre el modelo productivista que se había establecido en el siglo XIX y que se pulió en el siglo XX como industria organizada siguiendo el modelo consumista; el fordismo, que había conducido esta organización productivista-consumista a una especie de perfección, estaba fundamentado a la vez en el trabajo en cadena y en los medios de comunicación. Rompiendo con una edad dominada por las industrias culturales analógicas, el nuevo mundo industrial es lo que emerge a través de lo que se ha llamado sucesivamente la "sociedad de la información", la "sociedad del saber", la "industria del conocimiento" y la "economía de lo inmaterial". Que estos calificativos sean adecuados o no, es una amplísima pregunta que permanece abierta y sobre la que me he expresado en otra ocasión ${ }^{1}$.

Mi tercera convicción es que la superación del modelo productivista-consumista — que encuentra de ahora en adelante por todas partes sus propios límites sistémicos, lo que confirma las tesis que René Passet había enunciado hace veinticinco años (Passet, 1979) - tiene que ver de manera esencial con la aparición de un nuevo tipo de tecnologías relacionales. Las tecnologías relacionales aparecieron en el seno del estadio más reciente de la sociedad productivista-consumista, conduciendo a lo que Jeremy Rifkin llamó el "capitalismo cultural". Pero ellas son portadoras de un potencial relacional que rompe con la organización productivista-consumista en la medida en que esta reposa en una oposición funcional entre las dos instancias que la fundan: el productor y el consumidor.

La transformación de los modos de vida que se puso en funcionamiento desde comienzos del siglo XX con las public relations, que debían conducir a lo que luego de la Segunda Guerra Mundial se llamó marketing, constituirá una verdadera factura de las existencias individuales y colectivas por parte de las industrias de servicios, cuyas primeras y principales fueron las industrias culturales.

En el modelo de Jeremy Rifkin, los medios masivos explotan tecnologías relacionales analógicas que captan el tiempo de conciencia "proveyéndole" tiempo de experiencia estandarizada (como programa de televisión o de radio, pero también como industrias del turismo y del "descanso", lo que LeroiGourhan preveía y teorizaba desde $1965^{2}$ ). Pero las tecnologías relacionales

Sobre lo inmaterial, ver L'économie de l'hypermatériel et psychopouvoir (Stiegler, 2008a) y, sobre lo que François Fillon llamó la "batalla de la inteligencia", ver De la jeunesse et des générations (Stiegler, 2008b).

2 Cfr. El Gesto y la palabra (Leroi-Gourhan, 1971, pp. 346-349). 
típicas del siglo XXI ofrecen tiempo de conexión que es también tiempo de interacción, lo que constituye a este respecto un nuevo tipo de experiencia tecno-lógicamente relacional.

\section{El consumismo del modelo productivista}

El capitalismo está intrínsecamente ligado a la industria, y con muchísima mayor razón cuando se vuelve "cultural", incluso si hay un precapitalismo o un protocapitalismo que, analiza Max Weber, no es aún industrial. En desquite, este protocapitalismo es el que instala ya una división de los papeles sociales completamente nueva, y que será típica del capitalismo industrial, por donde se inventan las figuras del consumidor y del productor tal como se distinguen y se oponen funcional y relacionalmente. Weber, que describe al emprendedor del siglo XVIII que emerge en Pensilvania, muestra cómo ese protocapitalista se asigna por tarea separar la producción y el consumo, a la vez que los pone en relación, monopolizando para ello esta relación y poniéndose así en posición de intermediario.

Simplemente ocurrió esto: imaginemos un joven, hijo de una de las familias de empresarios residentes en la ciudad. Un buen día decidiría ir al campo para seleccionar con esmero los tejedores que le eran necesarios, los sometiría gradualmente bajo su dependencia y control, en pocas palabras, los transformaría de campesinos en obreros. A un tiempo, se encargaría personalmente de las transacciones, entablando contacto directo con los compradores al menudeo. Asimismo, se encargaría de manera directa de atraerse una nueva clientela, realizaría viajes cada año por lo menos y trataría, especialmente, de que la calidad de los artículos respondiera a la necesidad y al deseo de los compradores, aprendiendo así a "adaptarlos al gusto" de cada quien, comenzando por aplicar el principio de: "a precio barato mayor consumo". Llegando aquí, volvería a repetirse el fatal desenlace de todo proceso de "racionalización": el que no encaja el paso queda eliminado (Weber, 1981, p. 41) ${ }^{3}$.

Esta nueva organización de la sociedad que forma los lineamientos del productivismo y del consumismo, separando y oponiendo a los productores y a los consumidores, es lo que el capitalismo maquínico del siglo XIX va a sistematizar y a generalizar, pero buscando ante todo optimizar la productividad por medio de las inversiones en la innovación y la realización de economías de escala. Las ganancias de productividad van a ser tan importantes y rápidas que van a crear bien pronto una amenaza de superproducción.

Comenté este texto en Mécréance et discrédit 1. La Décadence des démocraties industrielles (Stiegler, 2004b, p. 102). 
Es por esto que el siglo XX, desde el nacimiento de lo que va a ser pensado como lo que llamamos hoy el design, el capitalismo va a dedicarse mucho más a formar mercados y, para ello, a captar el deseo de los consumidores para darle salida a su producción. Si el gran pensador de la sociedad industrial productivista salida del siglo XIX es — con Marx - Frederik Taylor, teórico de la organización científica del trabajo y autor de The Principles of scientific Management, el siglo XX está mucho más dominado por el pensamiento del marketing, cuya primera gran figura es la del sobrino de Freud, Edward Bernays. El marketing es una teoría de las relaciones, y se presenta ante todo como tecnología de las public relations ${ }^{4}$. Edward Bernays publica Crystallizing Public Opinion en 1923, en el momento mismo en que la Bauhaus trata de pensar nuevas relaciones entre el arte y la industria. Pero es con Ernest Dichter y Louis Cheskin, después de la Segunda Guerra Mundial, que aparece el marketing tal como lo conocemos. El objetivo ya no es formar y explotar productores, sino controlar comportamientos de consumidores por medio del desarrollo de psicotecnologías que tienden a liquidar la noopolítica propuesta desde Ferry por las instituciones de programas.

Un artículo de la agencia de publicidad MacGraw-Hill, publicado por la revista Advertising Age el 24 de octubre de 1955, se dirige así a los productores estadounidenses:

En tanto que nación, somos ya tan ricos que los consumidores no tienen ninguna necesidad de comprar una gran parte —quizás el $40 \%$ - de nuestra producción, y esta necesidad disminuirá progresivamente en el curso de los próximos años. Ahora bien, si los consumidores escogen no comprar una gran parte de la producción, no está lejana una fuerte depresión económica. (Calmann-Lévy, citado por Packard, 1958, p. 23)

Esta amenaza es, de hecho, una consecuencia concreta de la baja tendencial de la tasa de ganancia — que tiene como corolario el aumento del desempleo y la pauperización-; contra esta baja lucha el capitalismo no ha dejado de intensificar la innovación, lo que engendra entonces inevitablemente excedentes de producción y una obsolescencia crónica de los productos con respecto a la cual es necesario sostener cada vez más artificialmente el consumo, como lo muestra Vance Packard (1958):

Desde 1950, en momentos en que la superproducción amenazaba numerosos frentes, las preocupaciones de los dirigentes de las sociedades industriales sufrieron una modificación fundamental. La producción pasó para ellos a segundo plano. En lugar de pensar en fabricar, pensaran en vender. (p. 24)

La tesis presentada en este parágrafo está desarrollada en Prendre soin. De la jeunesse et des générations (Stiegler, 2008b). Allí mostré que, mucho más allá de lo que Michel Foucault llamó el biopoder, el siglo XX se caracteriza por la formación de un psicopoder cuyos órganos son el marketing y los medios de comunicación. 
La cuestión no es ya entonces, y en la actualidad mucho menos aún, controlar la población como máquina de producción (lo que Michel Foucault describió como el objetivo de lo que llamaba el biopoder), sino más bien como máquina de consumo; y el envite ya no es el biopoder sino el psicopoder como control y fabricación de motivaciones: "En las conferencias de industriales y de comerciantes, se habló de 'revolución del mercado', y se reflexionó mucho sobre los medios para 'estimular' a los compradores creándoles necesidades cuya existencia ellos ignoraban" (Packard, 1958, p. 24).

Es en este contexto en el que se desarrolla en los Estados Unidos la Investigación de los móviles, de la que Ernest Dichter es el principal representante. Plantea, en principio, que es necesario encontrar los medios de preacondicionar al cliente para comprar [los productos] "grabándole las características en el cerebro" (Packard, 1958, p. 28)

Es así como se emprende el proceso que conducirá a la destrucción del aparato psíquico juvenil y a la liquidación de las relaciones intergeneracionales. Para ello, tras las huellas de los primeros trabajos de Bernays (pero también de la cibernética aplicada a los condicionamientos de los equipamientos de las fortalezas volantes durante la Segunda Guerra Mundial), Dichter y Cheskin ponen el psicoanálisis al servicio del análisis sistemático de los mercados. Es así como invitan a los industriales a distinguir entre tres dimensiones en el aparato psíquico de sus clientes:

1. la consciencia, que no es manipulable;

2. el preconsciente, que los investigadores norteamericanos llaman con más gusto subconsciente;

3. el inconsciente, que alberga fuerzas, las pulsiones, que es posible manipular vía el preconsciente.

Vance Packard (1958) puede, desde entonces, escribir que "la exploración de nuestras actitudes con respecto a los productos, a los niveles dos y tres, es ahora conocida, en tanto que ciencia nueva, bajo el nombre de análisis o de investigación de móviles" (Packard, 1958).

\section{La superación del modelo productivista-consumista}

A comienzos del siglo XXI, al que acabamos de entrar al encuentro de tantas sorpresas, el sentimiento dominante es el de una gran perturbación, mucho más allá de la sola fecha del 11 de septiembre que, en una sola jornada, conmovió e hizo temblar el mundo por mucho tiempo. Sin embargo, no es ciertamente el

Packard cita aquí el New York World-Telegram and Sun. 
terrorismo el que constituye el hecho más conmovedor de este comienzo de siglo que es también un nuevo milenio; el potencial de desestabilización cuyos efectos sentimos que aumentan procede de una mutación sistémica mucho más profunda; y yo sostengo que ella nos ha de llevar a un tercer período de las sociedades industriales.

A este respecto, nuestra primera hipótesis común — digo común a la Escuela Superior de Creación Industrial (ESCI), al polo de competitividad Cap Digital y al Instituto de Investigaciones y de Innovación (IRI) del Centro Pompidou- es que entramos en un tercer período del capitalismo industrial donde la oposición producción/consumo tiende a volverse secundaria, es decir, ya no es portadora del dinamismo de este sistema dinámico que es el capitalismo industrial.

Ciertamente, esto no significa que esta división de los papeles en las relaciones sociales vaya a desaparecer (es muy verosímil que la industria nuclear, por ejemplo, vaya a permanecer aún durante mucho tiempo estructurada por esta oposición funcional). Pero, además de que la actividad de concepción de la producción nuclear será quizás conducida a evolucionar sensiblemente bajo la influencia de las tecnologías relacionales digitales, y en una relación nueva con su medio social, esto significa que las zonas de actividad económica e industrial por donde el sistema va a reconfigurarse serán estructuradas por nuevos tipos de relaciones entre los diferentes actores de la sociedad que se ha vuelto hiperindustrial e hipermaterial (Stiegler, 2008, p. 111).

Las nuevas relaciones industriales que instauran nuevas relaciones sociales en la sociedad hiperindustrial e hipermaterial serán cada vez más del orden de la contribución, por donde los actores tenderán a diluir la frontera muy tajante que separaba a los productores de los consumidores de manera, por lo demás, muy homogénea con los principios de la división industrial del trabajo que había conducido a la patología social de lo que Georges Friedmann llamaba el "trabajo en migajas" y luego a aquella que conocemos de ahora en adelante como consumo adictivo, que engendra su lote de miserias económicas, medioambientales, psicológicas y simbólicas.

Las nuevas relaciones industriales que instauran estas relaciones sociales contributivas se han hecho posibles por las tecnologías de la colaboración. La figura del contribuyente, entre los cuales el aficionado es en el mundo del arte y de la cultura una ocurrencia específica que le interesa evidentemente mucho al Centro Pompidou, y que nosotros estudiamos muy de cerca en el seno del Instituto de Investigación y de Innovación, es portadora de lo que André Gorz (1991) llamaba metamorfosis del trabajo. Pero lo que le concierne al trabajo, le concierne también a la vida por fuera del trabajo, y en realidad esta separación se vuelve cada vez más vaga desde que la oposición entre producción y consumo parece detenerse. 
Nuestra segunda hipótesis común es que la renovación de la figura del aficionado y la emergencia correlativa de la economía de la contribución han sido posibles a la vez por un poderoso deseo de la población, y en particular de la juventud, que ya no quiere contentarse con consumir, y por el despliegue de las tecnologías relacionales digitales que rompen la oposición entre producción y consumo al ofrecer funciones tanto de autoproducción como de indización sobre la web, donde se tejen nuevos tipos de redes que se llaman "sociales". Pensamos que la concretización y la cristalización sistémica de esta evolución conducirán a una economía industrial de la contribución.

Se puede y se debe evidentemente imaginar economías de la contribución que no reposen en la figura del aficionado. Sin embargo, es sobre todo a través de las prácticas culturales y las nuevas figuras del aficionado que ellas se engendran en el nuevo entorno relacional — a la vez bajo la forma a veces demasiado sabia que constituye la cultura del hacker, y en el sentido en que el intercambio de ficheros musicales constituye un embrión de práctica musical amateur como expresión de una elección y de un acto de administrar samples-, que se desprende el ideal-tipo de lo que es el contribuyente, haciendo emerger una economía de la contribución que nadie ha querido y que, en realidad, contraría los intereses de todos aquellos que querrían proteger su capacidad de querer, es decir, su poder.

Siempre es así como se hacen las grandes transformaciones, "en las espaldas de la conciencia", decía Hegel, y "con paso de paloma", añadía Nietzsche. Retengamos aquí que el mundo de la cultura se encuentra, de hecho, vuelto a poner en posición de vanguardia de la sociedad industrial que se inventa.

\section{La innovación ascendente, una nueva época del design}

Si vivimos en una sociedad más industrial que nunca, y si la economía industrial de mañana ya no reposará sobre la oposición funcional de la producción y del consumo inducida por la división industrial del trabajo, sino sobre un dispositivo de contribución que ya no opone esos papeles -oposición que condujo a la descomposición de la sociedad como trabajo en migajas y consumo adictivo destructor de las relaciones sociales-, sino que compone, por el contrario, verdaderas relaciones, será preciso inventar otra secuencia y otro tipo de relación entre el design y las otras funciones de desarrollo de productos industriales.

El design aparece en el segundo período del capitalismo, período que está esencialmente fundamentado en y por el control y la masificación de los comportamientos del consumidor. Mi tesis es que, en la época de la tercera forma de organización industrial de la sociedad, es necesario inventar una nueva relación entre ese design y el medio contributivo que debería darse 
como primera tarea "design-ar", contribuyendo él mismo a la confirmación y a la concepción de su emergencia.

Hacer un design en la época de la contribución, en la época de un modelo económico e industrial contributivo, del que las nuevas figuras de los aficionados deberían formar la vanguardia con el mundo artístico, es ante todo compartir la capacidad de design-ar, de concebir con los que antaño fueron llamados clientes, y que, al volverse contribuyentes, participan en la formación de un bucle del que se trata de convertirlo en una espiral fecunda más que en un círculo vicioso.

Esto supone ante todo rebasar la secuencia lineal que va de la concepción en sentido amplio (desde la investigación hasta el design, pasando por la investigación y el desarrollo, la ergonomía, etc.) a la distribución. No se trata simplemente de modificar bien en profundidad la división del trabajo y su organización, es decir, el management; se trata de cambiar por completo la naturaleza misma de las relaciones entre todos los actores directos e indirectos del hecho industrial total; y ya lo veremos con Yann Moulier Boutang cuando coloque de alguna manera las externalidades en el centro.

Ahora bien, lo que llamo el hecho industrial total es lo que debe dar cuenta del sitio de los beneficiarios del mundo industrial así como de sus víctimas. En el mundo industrial, como en todos los mundos humanos sin duda, pero quizás más brutal, constante y general que en toda otra forma de organización social, hay siempre víctimas; hay "roturas", transformaciones, es decir, destrucción que, incluso si es creadora (como lo dice Joseph Schumpeter), es ante todo destrucción, y produce entonces víctimas al destruir tanto hombres como cosas y entornos: aquello que se llama "externalidades". Ahora bien, en una "economía contributiva", ellas vendrían hacia el centro, y ahí estaría quizás para el design su más grande novedad.

\section{La nueva praxis y su utopía}

La figura del aficionado, del que he planteado que constituye el ideal-tipo del contribuyente (en el sentido en que Weber habla de un ideal tipo intrínsecamente utópico, que no existe pues de hecho, que es una idealidad dinámica organizadora de un medio social y que permite precisamente aclarar y describir su dinámica), es una figura de la economía libidinal perfectamente realizada, y tal que reposa ella misma, precisamente, en un proceso de idealización (que se traduce en el aparato psíquico en lo que Freud llama el ideal del yo).

La libido es lo que, como energía constituida por su economía y su puesta en reserva, transforma la satisfacción de las pulsiones, por esencia asociales, en un acto social que conduce en sus formas más socializadas a lo que se llama la 
sublimación. Y el aficionado, que es una figura ideal de esta sublimación ella misma idealizadora, es el que puede acceder a lo que se llama lo sublime.

El amateur es el que ama en este sentido, en el sentido en que, como todo amante, está claramente más poseído por lo que ama de lo que él mismo lo posee. Así mismo, la economía de la contribución no es tanto una economía de la posesión (y por tanto de la propiedad), sino una economía de la implicación y de la circulación de una energía libidinal que está engendrada por el intercambio en el curso del cual se produce una acumulación distribuida más que captada. Es pues una economía no-rival, como lo dice Michel Gensollen (2004), "donde el valor se sitúa mayoritariamente en la elaboración de las formas más bien que en su reproducción industrial".

En el siglo XX se situó una economía libidinal industrial, es decir, una organización industrial de la captación de la libido como energía que se ha tratado desde entonces, precisamente con Bernays, de desviar hacia la mercancía. Aquí mismo he sostenido, hace tres años ${ }^{6}$, que ese capitalismo industrial del siglo XX había explotado la figura del consumidor a tal punto que había terminado por destruir su libido, y que el design estaba en gran medida inscrito en la localización de ese sistema.

Me gustaría hoy encadenar con ese análisis y añadirle aquí que la nueva figura del aficionado como ideal tipo de una economía de la contribución, se afirma como una especie de relevo de esta negatividad (en el sentido del Aufhebung de Hegel); es esta posibilidad de relevo "relève» la que constituye la tarea del nuevo design como un desafío que se trata pues de aceptar "relever».

La formación del contribuidor como actor económico es, en efecto, en tanto que su ideal tipo es el amateur, una reafirmación de la libido y una reinvención de su economía que termina por ser arruinada por las técnicas de captación y de desvío del marketing ${ }^{7}$ (catástrofe que produce desmotivación, disgusto y adicción); el contribuidor es el que reafirma la necesidad de construir una economía libidinal durable, si puedo decir así, y que la construye él mismo. No espera que la sociedad industrial la construya en su lugar.

La formación de una figura tal es una situación producida por la sociedad; ella no es producida por iniciativa del mundo industrial. En desquite, esta iniciativa de la sociedad, que no es una iniciativa del mundo industrial, se ha apoderado de una proposición del mundo industrial, de una propuesta tecnológica engendrada

En el curso del coloquio Pensar el design que fue luego publicado en las ediciones de la Regard, bajo la dirección de Brigitte Flamand.

7 Traté de describir las causas y los efectos de esta destrucción en Mécréance et discrédit 1. La Décadence des démocraties industrielles. y en De la misère symbolique: Tome 1. L'époque hyperindustrielle. 
por el desarrollo de las telecomunicaciones, de la informática, del audiovisual y de lo que, a través de la digitalización, se ha puesto a converger en una nueva red que constituye un medio sociotécnico asociado, es decir, un medio donde todos los actores son participativos y donde el proceso de lo que se realiza allá es el estadio más reciente de un proceso de gramatización (es decir, de reproducción y discretización cuyo caso más conocido es la gramatización del lenguaje por la escritura); el proceso de gramatización de las relaciones sociales en tanto que tales.

El desarrollo de la informática, el audiovisual y las telecomunicaciones que convergen en la digitalización constituye un nuevo estadio de la reproductibilidad tal y como ella se vuelve accesible a casi todos y a costo casi nulo, lo que permite súbitamente a toda suerte de actores acceder a funciones que no eran hasta entonces accesibles más que a profesionales. Es así como se ve aparecer en la vida cotidiana de las personas toda suerte de funciones técnicas hasta hace poco muy sofisticadas, como por ejemplo la gestión de las indizaciones por motores de búsqueda y la realización de peticiones sobre bases de datos; hacer una búsqueda en Google es hacer una tal indagación, y el hombre ordinario del país hiperindustrializado efectúa a menudo muchas de ellas en la misma jornada.

Hace muy poco tiempo que todo esto se volvió posible. Hace veinte años, cuando presenté al Centro Pompidou la exposición Memorias del futuro, buscar un ítem en una base de datos, utilizar o alimentar un dispositivo de indización, anotar e inscribir datos, etc., eran funciones muy especializadas, muy "puntudas", como se estilaba decir entonces con una palabra un poco ridícula; esas operaciones estaban reservadas a los profesionales de los nuevos oficios. Insisto en la indización, pero se necesitaría hablar también de la autoproducción audiovisual que está en vías de invadir la red digital precisamente desde que se concibió un sistema rudimentario pero eficiente de alojamiento y de distribución indizada de esos videos autoproducidos.

Este devenir general por donde se diseminan nuevas competencias en la sociedad, precisamente en la medida en que ellas se vuelven saberes de aficionados, escapa a las corporaciones de los diversos profesionales de muchas profesiones; en particular de los saberes informáticos, que se difunden de tal manera que se ve cómo se desarrolla toda suerte de competencias que no han sido anticipadas por la UTC, por la Ensci, por la Escuela Nacional Superior de Telecomunicaciones (ENST) ni por ninguna escuela, porque esos saberes se forman más pronto de lo que se escribe o se concibe un curso, y porque son cada vez más el fruto de la actividad de apasionados y de amateurs en ese sentido, en el sentido de lo que Pekka Himanen llama hackers. 
¿Qué es un hacker en el fondo, y qué hay de positivamente subversivo en esta figura? Yo concibo esta figura y su positividad por su capacidad de apropiarse de la oferta tecnológica e industrial sin conformarse con las prescripciones del marketing de esa oferta, queridos por los planes de desarrollo concebidos por la industria. Y ello ocurre así porque lo que ponen en funcionamiento estos actores, que ya no son ni consumidores ni clientes, que no son usos, como lo creían los industriales que querrían a través de esos usos controlar el devenir de su producto; son prácticas sociales, individuales y colectivas de una gran complejidad y que tienen que ver con lo que se llamaba antaño una praxis, pero en un sentido muy renovado y revisitado.

\section{La hipermaterialidad de las tecnologías relacionales y transformacionales}

La economía de la contribución emerge al estadio más reciente del proceso de gramatización, como discretización, formalización, reproducción y reticulación tecnológica de las relaciones. Pero esta gramatización se opera igualmente a nivel del viviente y de la materia inerte con las biotecnologías y las nanotecnologías. Creo que esta conjugación de factores plantea la cuestión de lo que llamé la hipermateria y lo hipermaterial. Aparece así que la época de la economía de la contribución es también la de la economía de lo hipermaterial, y no de lo inmaterial, como tan a menudo se lo pretende.

En momentos en que vivimos la enorme transformación que designo aquí con el nombre de sociedad hiperindustrial, que echa por tierra un sistema que se instaló hace más de dos siglos y que reposaba en la distinción productor/ consumidor, lo que lleva a Pekka Himanen a sostener que rompemos así con la ética protestante del capitalismo, es decir, con el espíritu del capitalismo según Max Weber, aparece otro elemento muy importante de ruptura que concierne a la materia y a la forma y, más allá o más acá de la materia y de la forma, a la energía y la información, en el sentido que esas palabras tienen en física cuántica.

Lo que parecía constituir el zócalo de la sociedad industrial, el elemento que había permanecido sólido de esta sociedad a tal punto inestable, entra él también, en efecto, en transformación, a tal punto que para designar esta novedad se habla de tecnologías transformacionales. A pesar de la metaestabilidad muy fluida que no ha cesado de extenderse con la sociedad industrial, y como su carácter propio, pensamos hasta hace muy poco que esta metaestabilidad preservaba la "tierra firme" (si puedo decirlo); o, para ser más preciso, una permanencia de los datos de los que las ciencias de la naturaleza hacen sus objetos como física o como biología. Durante mucho tiempo, la ciencia se define planteando que, más allá de todas las transformaciones que afectan su objeto, este está constituido por características esenciales y, en esto, en y por la firmeza de su ser. 
Durante mucho tiempo, la confianza del hombre ordinario en la industria -que ha hecho de esas ciencias tecnociencias- habrá reposado sobre la idea de que esas ciencias mantenían firme un objeto firmemente constituido, y que forma precisamente por esto un ser, es decir, la unidad idéntica de lo que no deviene. Es este ser estable el que fundaba la solidez de un zócalo de estabilidad que soporta y contiene toda suerte de inestabilidades; durante mucho tiempo se creyó que no era transformable, como hay cosas de las que se dice que no son negociables o que no son calculables. Ahora bien, todo esto también se vuelve transformable y metaestable, por no decir potencialmente inestable. A este respecto, las tecnologías transformacionables constituyen una hipertransformación; y esta es típica de la sociedad hiperindustrial, mientras que las tecnologías relacionales participan en un inmenso proceso de transformación social.

Este dato - lo que yo llamo pues hipertransformación- concerniente a toda la actividad industrial (tanto la de los productores y de los consumidores como la de los contribuidores que, en tanto que pares, escapan a esta dicotomía funcional), concierne en primer lugar al designador en tanto que, hasta entonces, él estaba obligado a trabajar con formas y con materias o materiales, cuando el trabajo del designador reposaba en la diferencia entre la forma y la materia. Ahora bien, ya no es posible pensar lo real hiperindustrial oponiendo tales categorías; y como lo escribe Gilbert Simondon, se precisa superar el esquema hilemórfico, es decir, el que opone la hylè (materia) a la morphè (forma) para razonar en términos de procesos de individuación, es decir, de transformación.

La hipermateria es lo que se transforma en el curso de un tal proceso donde no es pertinente distinguir la materia de su forma; ella es aquí siempre ya una forma. Y es esto lo que hace aparecer muy manifiestamente la tecnología de la "materia" nanométricamente manipulada y dramatizada; las nanoestructuras son siempre ya formas o, más bien y más precisamente, procesos tramados por la pareja energía/información que constituye ya la cuestión de la hipermateria en la mecánica cuántica, mucho antes de las nanociencias y las nanotecnologías. Sin embargo, estas últimas hacen aparecer esta cuestión de manera muy singular y como asunto de una transformación tal que ella desestabiliza lo que se presentaba hasta entonces como el zócalo firme del devenir industrial.

Es necesario, sin embargo, hablar de hipermaterialidad igualmente en el sentido en que vivimos en la sociedad hipermaterializada (constituida por una materia que da una forma, como información, y a partir de una energía) de tal suerte que prolifere allí lo hipermaterial que se inmiscuye en todas las relaciones, como teléfono celular, computador, Ipod, chip RFID, microtecnologías y un día como nanotecnologías compatibilizadas con las biotecnologías. 
Lo que se llama inmaterial, es decir, la información, no es pues nunca inmaterial; la menor señal es siempre material, requiere solo algunos electrones. Hay siempre materia en la forma como hay siempre forma en la materia. Los trenes binarios de información, que traman cada vez más nuestras relaciones, y que son ya hipermateria, necesitan lo que se llama todo ese "material" que es en realidad de lo hipermaterial. Ahora bien, este hipermaterial que es microtecnológico, está él mismo en vías de mutar; pronto se reconfigurará a través del desarrollo de las tecnologías transformacionales. Y es aquello con lo que los designadores van, de acá en adelante, a tener que componer.

Este proceso es hipertransformacional porque transforma las condiciones mismas de la transformación; la industria es, por otra parte, desde su origen y en su hecho más general, un modo de la transformación industrial por la reproductibilidad. El hombre es él mismo esencialmente un transformador —en tanto que técnico- desde su origen y en su hecho más general. Lo que caracteriza la transformación industrial es que ella consiste en una transformación que se hace por estandarización de las condiciones de transformación, lo que es una modalidad particular de la gramatización.

Si tratamos en este momento de hacer la síntesis de todo lo que ha sido dicho hasta aquí, vemos que asistimos a una transformación de las condiciones industriales de transformación por el hecho de la aparición de, por una parte, las tecnologías transformacionales y, por otra parte, una modalidad de distribución de los papeles entre los transformadores, donde el destinatario de las transformaciones ya no es el que - como lo era el cliente-, sufre todas esas transformaciones, sino de las que él se vuelve también, por el contrario, un destinador, es decir, un transformador. La apuesta del nuevo mundo industrial es en esto la invención de un circuito de transformación generalizada, o más bien de un conjunto reticular de circuitos que cumplen esta transformación generalizada. Las concepción de un tal conjunto es el envite de una economía política de la contribución, y tal es también, yo creo, el tema de nuestros encuentros.

\section{Referencias}

Gensollen, M. (2004). Economie non-rivale et communautés d'information. Réseaux, (124).

Gorz, A. (1991). Metamorfosis del trabajo. Búsqueda de sentido. Crítica de la razón económica. Sistema. Leroi-Gourhan, A. (1971). El Gesto y la palabra. Universidad Central de Venezuela.

Packard, V. (1958). La Persuasion clandestine. Calmann-Lévy.

Passet. (1979). Lıéconomique et le vivant. Payot.

Stiegler, B. (2004). De la misère symbolique: Tome 1. Liépoque hyperindustrielle. Galilée.

Stiegler, B. (2004b). Mécréance et discrédit 1. La Décadence des démocraties industrielles. Galilée. 
Stiegler, B. (2008). Industrie relationnelle et économie de la contribution. En B. Stiegler (dir.). Le «design» de nos existences: à liépoque de linnovation ascendante (pp. 20-42). Fayard.

Stiegler, B. (2008a). Liéconomie de lihypermatériel et psychopouvoir. Mille et une nuits.

Stiegler, B. (2008b). Prendre soin, de la jeunesse et des générations. Flammarion.

Weber, M. (1981). La ética protestante y el espíritu del capitalismo. Premia. 